\title{
OPAQUE ARTHROGRAPHY OF THE KNEE JOINT
}

\author{
BY \\ A. E. JOWETT \\ From Musgrove Park Hospital, Taunton
}

There are occasions when radio arthrography of the knee joint is of value, both as an aid to diagnosis in obscure cases and for assessing the results of treatment. Cullen and Chance (1943) and Somerville (1946) have shown the value of air arthrography in cases of lesions of the menisci, and before that Lagergren (1934) used a radio-opaque medium for the same purpose; but it is felt that opaque arthrography should have a wider application than this as it will give useful information, sometimes of a more precise nature than can be obtained in any other way, as to the state of the articular cartilages, the synovial membrane, the presence of non-opaque bodies within the joint, or the relationship of an opaque body to the synovial cavity. Opaque arthrography has been used in other joints, notably in the hip joint by Sievers (1926-7), Leveuf and Bertrand (1937), and Severin (1939), in cases of congenital dislocation.

The first arthrogram in this series was taken at the Princess Elizabeth Hospital, Exeter, in March 1946, and since that time a further forty-seven knees (forty-four patients) have been examined in this way at the Princess Elizabeth Orthopaedic Hospital, Exeter, Mount Gold Hospital, Plymouth, and Musgrove Park Hospital, Taunton. A preliminary report on sixteen cases was made at the British Orthopaedic Association Spring Meeting at Exeter in May 1947.

\section{Opaque-Media}

The opaque media used have been two proprietary preparations of 3,5 , di iodo-4-pyridone- $\mathrm{N}$-acetic aciddiethanolamine, commonly known as "Diodone" and originally used for excretion urography. The preparations used are "Pylumbrin" (Boots) and Diodrast (Winthrop), which consist of a 35 per cent. solution of the drug in water, giving a neutral and stable solution which can be sterilized by heat. The solution is much heavier than synovial fluid and the two do not readily mix without shaking. A 17.5 per cent. solution has been used in some cases, but, owing to its rapid absorption the time during which satisfactory radiographs can be obtained is short.

Dosage.-The dosage is limited rather by the capacity of the knee joint than by the toxicity of the opaque medium. Normally up to $30 \mathrm{c.cm}$. may be given intravenously to an adult, whereas it has not been found necessary to inject more than $12 \mathrm{c.cm}$. into a knee joint, $9 \mathrm{c.cm}$. being usual for an adult. In children the dosage is proportionately reduced. In one patient (Fig. 16), the knee joint did not communicate with the suprapatellar pouch; this was not known at the time of injection and $9 \mathrm{c} . \mathrm{cm}$. of medium were injected. This seemed to have been rather too much for a joint thus reduced in capacity, for the knee was rather tense for three days afterwards, but it recovered completely in a few days.

After-Effects.-The injection is often followed by a small effusion with little or no pain; this subsides in a few days : in no case has there been any ill-effect lasting beyond a week. Occasionally a joint which formerly contained an effusion has been clinically and, in the patient's opinion, better than before arthrography. Lagergren (1934), using " Perabrodil" (Bayer), which is also a 35 per cent. solution of "Diodone ", carried out biopsies of synovial membrane in those cases subsequently submitted to arthrotomy. In no case was there histological evidence of irritation. This has been confirmed in two of the present cases.

"Diodone" is said occasionally to give rise to allergic phenomena, when given intravenously. No such phenomena have been seen in this series, but in allergic subjects, and particularly in those with an iodine sensitivity, the examination should be approached with caution.

\section{Method of Administration}

The method of administration has been as follows : After thorough skin preparation and local infiltration with 2 per cent. procaine solution, a needle of moderate bore is made to enter the knee joint : any excess of synovial fluid is aspirated from the joint and about $5 \mathrm{c.cm}$. of this is thoroughly mixed with the medium which is then injected into the joint. The needle is withdrawn and the puncture covered with a sterile dressing, which is loosely bandaged. The knee joint is then made to move through its full range a few times and the patient is taken to the $x$-ray department. The mixture of the drug with synovial fluid seems to help the diffusion of the medium throughout the joint, though there has been no delay of this in " dry " joints where such mixing has not been possible.

For two days afterwards, the patient is kept in bed, carrying out non-weight-bearing exercises.

\section{Radiographic Technique}

No specialized radiographic technique has been used. Antero-posterior lateral, and antero-posterior oblique 
views (with the limb medially and laterally rotated to forty-five degrees) are taken as a routine. A tangential view of the patello-femoral joint is taken, where indicated, as are also postero-anterior oblique views.

The drug is absorbed fairly rapidly, but good shadows are usually obtainable for fifteen minutes after injection. In the three cases tested the shadow due to the drug became indistinguishable between three and a half and four hours afterwards.

\section{Resuilts}

The normal antero-posterior view shows the suprapatellar pouch of normal size and shape : a thin and regular film of medium extends across the middle of the joint, outlining the cartilaginous covering of the bony surfaces: towards either side of the joint this film, which represents the true joint space, splits to enclose the menisci, which are triangular in section. The lateral meniscus is often bounded by a vertical shadow, which usually dips below the upper surface of the tibia : this is the synovial sheath of the popliteus. These characteristics are seen in Fig. 1, but the suprapatellar pouch is smaller than usual as a result of an old wound. A normal medial meniscus is also well seen in Fig. 8.

The normal lateral view (Fig. 2) shows the suprapatellar pouch of normal size and shape, the outline of the cartilaginous surface of the patella, and a film of medium covering the articular cartilage of the femur, which meets that covering the tibial joint surface and so outlines the infrapatellar pad of fat. The film of medium extends between the contiguous surfaces of the femur and tibia and splits again to extend over the back of the femoral and tibial condyles. Sometimes this posterior pouch of the joint is more bulky.

The oblique views are used mainly to demonstrate the menisci. The external oblique, taken in an antero-posterior direction, with the limb rotated outwards forty-five degrees (Fig. 3), shows the anterior end of the lateral meniscus and the posterior end of the medial meniscus. Both are triangular in section, with smooth surfaces. The internal oblique view (Fig. 4) shows the anterior end of the medial meniscus and the posterior end of the lateral meniscus : both are of normal appearance, the latter being bounded peripherally by a vertical film of medium, which is occupying the synovial sheath of the popliteus tendon.

Fig. 5, a tangential view of the patello-femoral joint, shows a smooth regular film of medium between articular cartilages of equal depth except at the medial end, where there is some irregularity, probably due to early arthritic change : the film of medium is denser than usual.

Figs. 6 and 7 are arthrograms of the knee of a boy of nearly seven years. The arthrograms show well the depth of cartilage which clothes the epiphyses of the femur and tibia and the patella at this age ; the defining of the cartilage gives a much more adult outline to the bone-ends. The medial meniscus is well seen, but the lateral meniscus is less definite in outline.

Fig. 8 shows a tear of the lateral meniscus, represented by a band of the medium traversing the shadow of the lateral meniscus, which contrasts well with the normal shadow of the medial meniscus. Unfortunately, it has not yet been possible to confirm this finding by operation, as the patient had a severe hysterical palsy of his hand which, it was considered, should have priority of treatment over his knee condition, which was causing him relatively slight disability.

Fig. 9 shows an oblique view of a medially rotated knee ; the normal triangular outline of the posterior end of the lateral meniscus is just visible ; the shadow of the anterior end of the medial meniscus is shortened and rounded. This finding was confirmed at operation, which revealed a "bucket-handle" tear of the medial meniscus, with displacement into the middle of the joint.

Figs. 10 to 13 illustrate a case of villous arthritis. Figs. 10 and 11 are of pre-operative films, showing a grossly enlarged suprapatellar pouch with much folding of the synovial membrane; some air which had inadvertently been allowed to get into the joint is seen in the posterior pouch. The upper part of the suprapatellar pouch had hardly been penetrated by the medium and was found at operation to have been almost completely separated from the joint by a synovial septum. Figs. 12 and 13 are of the films taken eleven months after synovectomy : the suprapatellar pouch is of normal size, though it still shows a tendency to folding of the synovial membrane. The lower part of the joint is seen to be normal and there is a good posterior pouch.

Fig. 14 illustrates a case of chronic fibrinous $\frac{D}{2}$ arthritis of the knee occurring late in the course of chronic infective polyarthritis. The arthrograms of show several films of opaque medium between $N$ translucent areas in the suprapatellar pouch, the N nature of which was not fully appreciated until ${ }_{\sigma}^{\mathcal{O}}$ synovectomy was carried out : the suprapatellar pouch was then found to contain several large masses of fibrinous material, which constituted the $\stackrel{\odot}{+}$ " filling defects" noted radiographically.

Figs. 15 and 16 are taken from a case of an old injury to the knee joint, which included a depressed fracture of the lateral tibial condyle and lesions of $\mathbb{Q}$

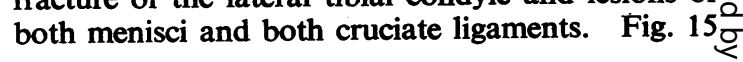




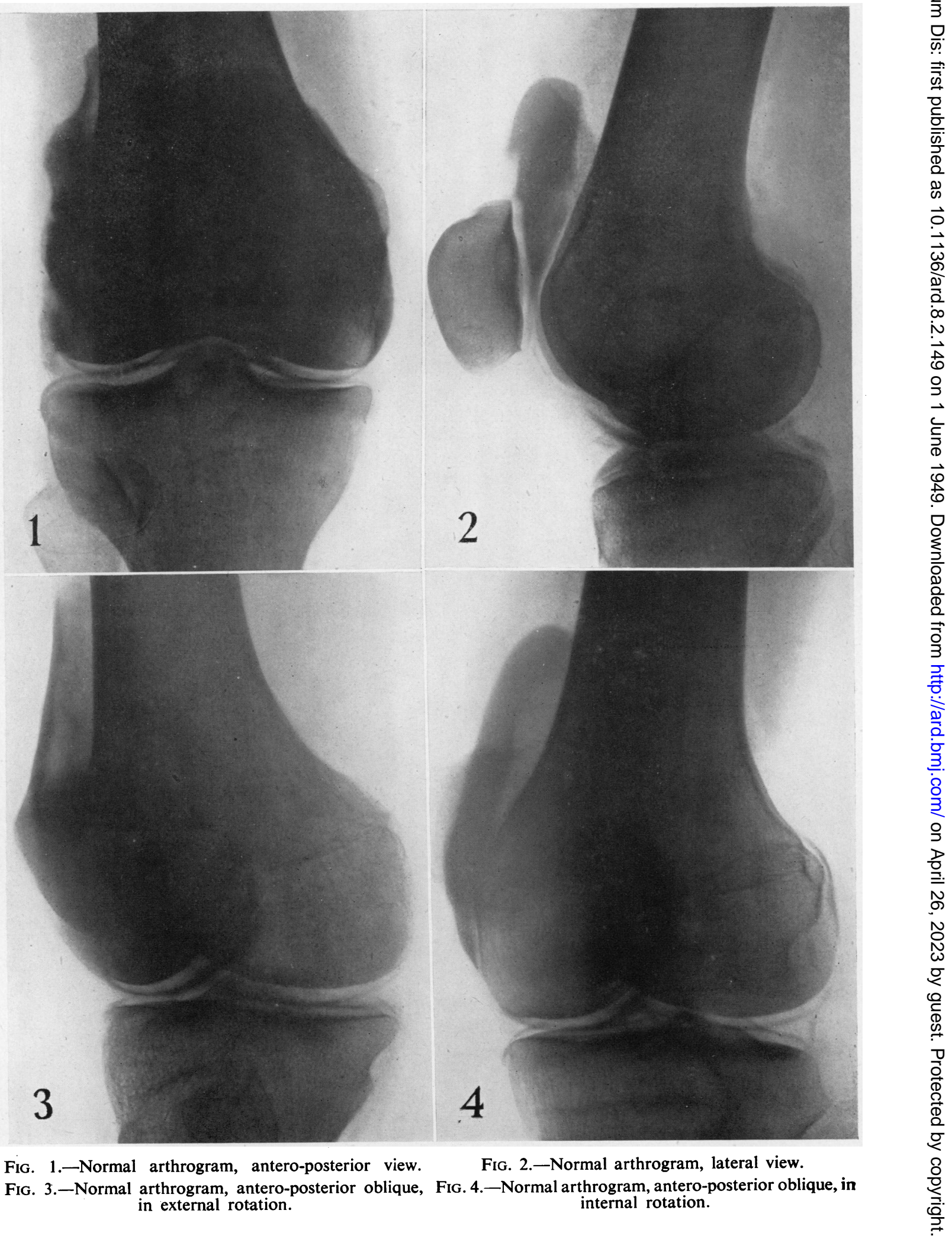




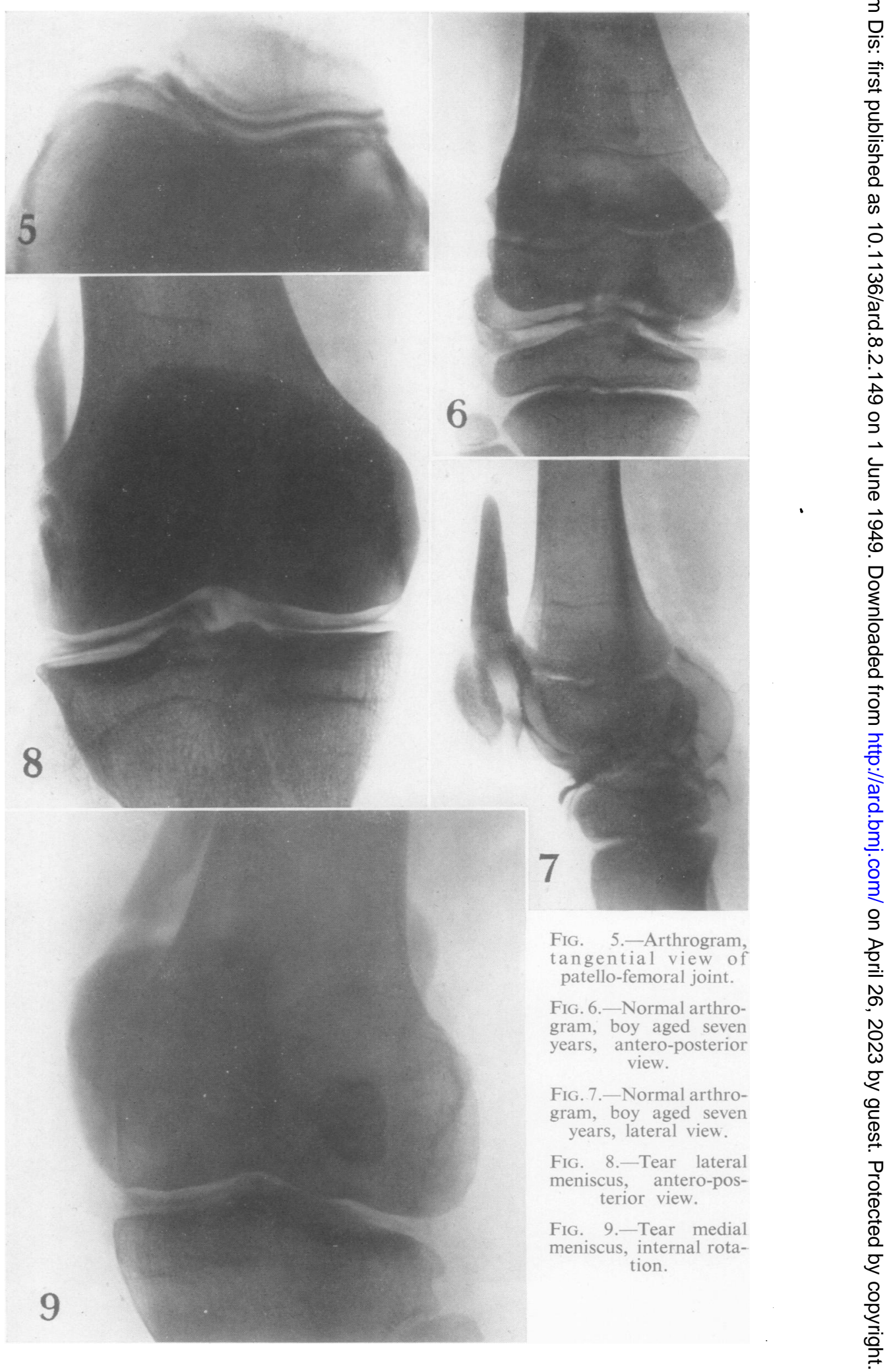




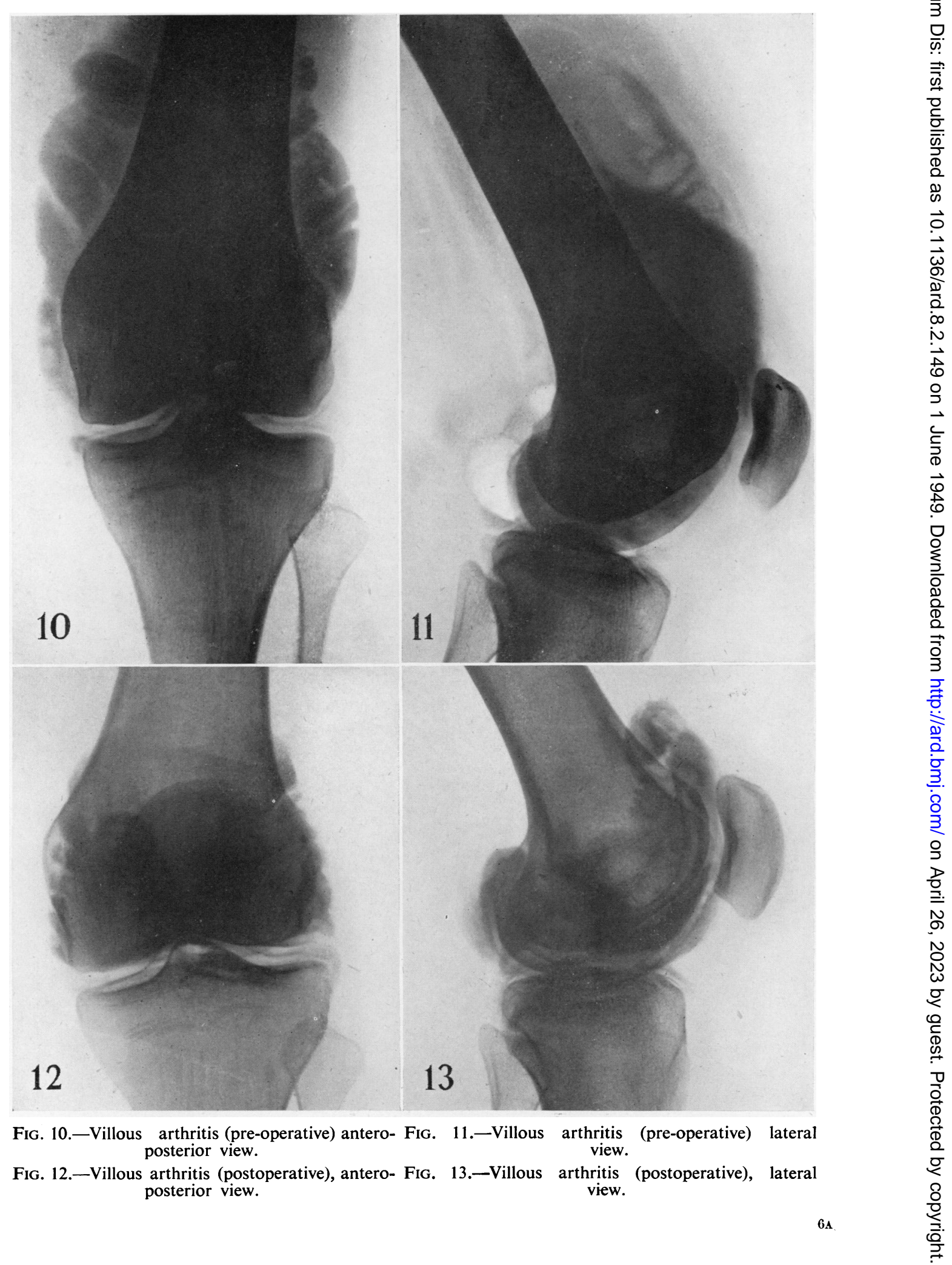




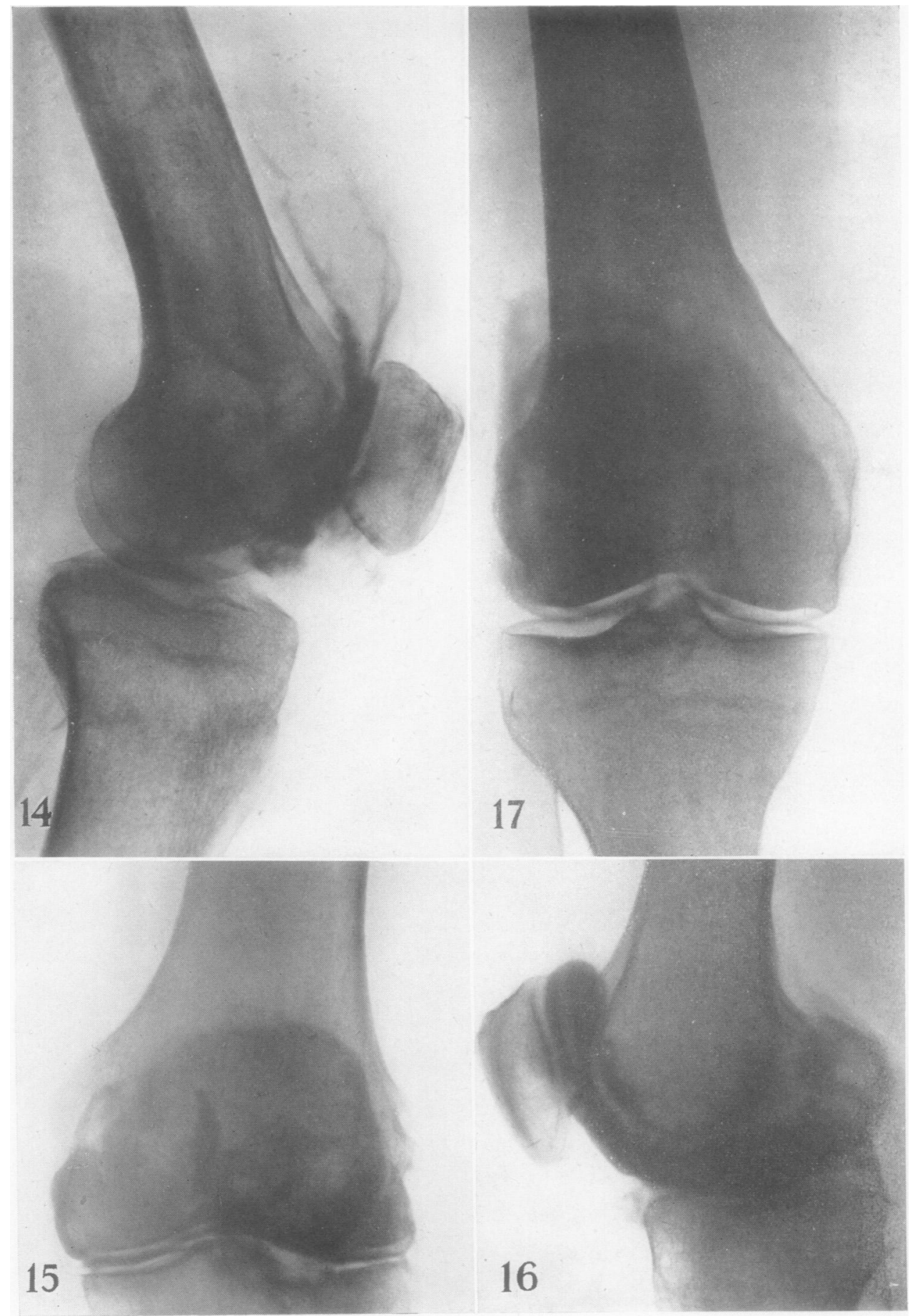

FIg. 14.-Chronic fibrinous arthritis, lateral FIG. 17.-Antero-posterior arthrogram of a view. case of early osteo-arthritis.

Fig. 15.-Old depressed fracture, lateral tibial FIG. 16.-Same case as Fig. 15, showing condyle, lateral view. absent supra-patellar pouch, lateral view. 
shows how a defect in the lateral tibial condyle has been filled in, probably by fibrous tissue, the actual joint surface, as defined by the opaque medium, being regular and smooth. The menisci had been removed at operation soon after the injury. Fig. 16 shows that the synovial lining of the knee joint does not extend for more than a quarter of an inch above the patella : a well-filled posterior pouch is seen.

Fig. 17 illustrates a case of early osteo-arthritis. Osteophytes, which are seen at the edges of both femoral and the medial tibial condyles, do not yet encroach upon the joint space as demonstrated by the film of opaque medium. The articular cartilage is elsewhere smooth and of normal thickness. The menisci appear normal. The suprapatellar pouch is small.

\section{Possibilities for the Future}

At present little is known about opaque arthrography of the knee, but it is expected that, as it becomes more widely used, improvements in technique and, particularly, in the interpretation of results will appear. It is a simple procedure, requiring no specialized apparatus or technique, and with proper care it appears to be safe. It is not, however, without its limitations, as in one recent case where a patient had a peripheral detachment of more than half of his medial meniscus, subsequently proved at operation, and yet no shadow suggestive of this was seen in the arthrograms.

\section{Summary}

The technique of opaque arthrography of the knee is described, and its wider employment than has been usual heretofore is suggested.

The appearances of normal and of some abnormal arthrograms are described and illustrated.

I am indebted to the staffs of the hospitals concerned for much help, and I wish particularly to thank Mr. Norman Capener who initiated this work and who has given continuous help and advice.

\section{REFERENCES}

Cullen, C. H., and Chance, G. Q. (1943). Brit. J. Surg., 30, 241 .

Lagergren, K. A. (1934). Acta Chirurg. Scand., 75, 485. Leveuf, J., and Bertrand, P. (1937). Presse Méd., 45, 437.

Severin, E. (1939). J. Bone J. Surg., 21, 304.

Sievers, R. (1926-7). Quoted by Severin.

Somerville, E. W. (1946). J. Bone Jt. Surg., 28, 451.

\section{Arthrographie Opaque de l'Articulation du Genou Résumé}

L'auteur décrit la technique de l'arthrographie opaque du genou, et pense qu'elle devrait être employée plus souvent qu'elle ne l'est actuellement. Il décrit et illustre les aspects des arthrographies normales et de quelques arthrographies anormales. 